\title{
Continuous Pharmaceutical Manufacture Using a Novel Wet Granulation Technique
}

\author{
J.P. K. SeVILle, M. WATERS
}

School of Engineering, University of Warwick, Coventry, England

E-mail: J.P.K.Seville@warwick.ac.uk (J.P. K. Seville)

Sci Pharm. 2010; 78: 543

doi:10.3797/scipharm.cespt.8.L03

\begin{abstract}
Almost alone among the process industries, the pharmaceutical industry has tended to rely on batch operations. This is now changing, driven by a need for better utilisation of resources, particularly clean space, and a demand for more closely controlled processes. Process Analytical Technology (PAT) demands that processes be controlled; this is much more easily engineered into continuous processes.

Wet granulation presents a particular problem, in that it has traditionally been carried out in a high-shear mixer-granulator and is not particularly well understood. What is known is that the mechanisms of nucleation, consolidation, growth and breakage occur more-or-less simultaneously in a high shear environment, and in a relatively uncontrolled way. In principle, it would be highly attractive to separate these basic mechanisms so that they occur within a continuous process in a controlled way.

This paper proposes the practical development of an overall process for continuous pharmaceutical manufacture, with consideration of process control, instrumentation, validation and real-time product release.
\end{abstract}

